\title{
The scaling of leaf area and mass: the cost of light interception increases with leaf size
}

\author{
Rubén Milla* and Peter B. Reich \\ Department of Forest Resources, University of Minnesota, 1530 Cleveland Avenue North, St Paul, MN 55108, USA
}

\begin{abstract}
For leaves, the light-capturing surface area per unit dry mass investment (specific leaf area, SLA) is a key trait from physiological, ecological and biophysical perspectives. To address whether SLA declines with leaf size, as hypothesized due to increasing costs of support in larger leaves, we compiled data on intraspecific variation in leaf dry mass (LM) and leaf surface area (LA) for 6334 leaves of 157 species. We used the power function $\mathrm{LM}=\alpha \mathrm{LA}^{\beta}$ to test whether, within each species, large leaves deploy less surface area per unit dry mass than small leaves. Comparing scaling exponents $(\beta)$ showed that more species had a statistically significant decrease in SLA as leaf size increased (61) than the opposite (7) and the average $\beta$ was significantly greater than 1 $\left(\beta_{\text {mean }}=1.10,95 \%\right.$ CI $\left.1.08-1.13\right)$. However, scaling exponents varied markedly from the few species that decreased to the many that increased SLA disproportionately fast as leaf size increased. This variation was unrelated to growth form, ecosystem of origin or climate. The average within-species tendency found here (allometric decrease of SLA with leaf size, averaging 13\%) is in accord with concurrent findings on global-scale trends among species, although the substantial scatter around the central tendency suggests that the leaf size dependency does not obligately shape SLA. Nonetheless, the generally greater mass per unit leaf area of larger than smaller leaves directly translates into a greater cost to build and maintain a unit of leaf area, which, all else being equal, should constrain the maximum leaf size displayed.
\end{abstract}

Keywords: leaf area; leaf mass; specific leaf area; allometry; scaling; power laws.

\section{INTRODUCTION}

The size of living organisms and their organs mediates a variety of biological processes (Peters 1983; Leonard \& Robertson 1994). Terrestrial plants are not an exception. Tree size influences gas exchange rates to such an extent that the decrease of water transport efficiency with size poses a limit to maximum tree height (Koch et al. 2004). At the organ level, size dependency may influence processes as disparate as the evolution of fruit size and dispersal system (Herrera 2002) or the partition of biomass to leaves, stems or roots (Enquist \& Niklas 2002). The scaling of leaf mass to leaf area is a particularly relevant relation because it directly impacts the specific leaf area (SLA), the amount of light-capturing surface area that is deployed with a given investment of dry mass. Given the direct relationship between SLA and light interception (Reich et al. 1998), SLA represents a potential revenue stream and hence is analogous to a potential rate of return on dry mass investment in terms of light capture (Westoby et al. 2000). Consequently, SLA is intimately connected to the resource use economy of the plant (Reich et al. 1997, 1998; Wright et al. 2004), its relationships with decomposers and herbivores (Cornelissen et al. 1999) and the way plant species replace each other during succession (Shipley et al. 2006). The understanding of size-related biophysical limitations of SLA is therefore of paramount relevance on physiological and ecological grounds.

\footnotetext{
* Author and address for correspondence: Área de Biodiversidad y Conservación, Universidad Rey Juan Carlos, c/Tulipán s/n., 28933 Móstoles, Madrid (Spain) (ruben.milla@gmail.com).
}

Electronic supplementary material is available at http://dx.doi.org/10. 1098/rspb.2007.0417 or via http://www.journals.royalsoc.ac.uk.
Arguments have been made both in favour of and against the idea that larger leaves should require proportional greater investment in support and structure. Some have proposed that mechanical support and dry mass investments per unit leaf area should scale positively with size to compensate for mechanical damage and gravity, since leaf laminas are cantilevered structures (Howland 1962; Grubb 1998). Alternatively, larger leaves may require less investment in support in general, because they reduce the number of leaves that have to be produced per shoot or plant to support a given leaf area (Givnish 1979). Additionally, across temperature gradients, size may decrease and density increase in acclimation to higher temperatures (Givnish 1987; Wright et al. 2005), and as a result leaf mass per unit area would decrease with increasing size.

The empirical evidence from published reports is inconclusive with respect to SLA and leaf size. Several studies have found that larger leaves need more specific support per unit leaf area than smaller ones measured as investment in petioles, lamina dry mass, twig size or venation stiffness (Shipley 1995; Grubb 1998; Gunn et al. 1999; Niklas 1999; Niinemets \& Kull 1999; Niinemets et al. 2006). In contrast, Niinemets \& Kull (1994) reported that in a comparison of 83 woody temperate species, SLA increased with leaf size. Ackerly \& Reich (1999) also showed a significant positive relationship of SLA with leaf size across an alternative set of 85 species of angiosperms.

Thus, there is no consensus among disparate studies regarding whether SLA declines, increases or remains constant with increasing leaf size. However, the studies described above pull together a variety of uncontrolled environmental and phylogenetic sources of variation, 


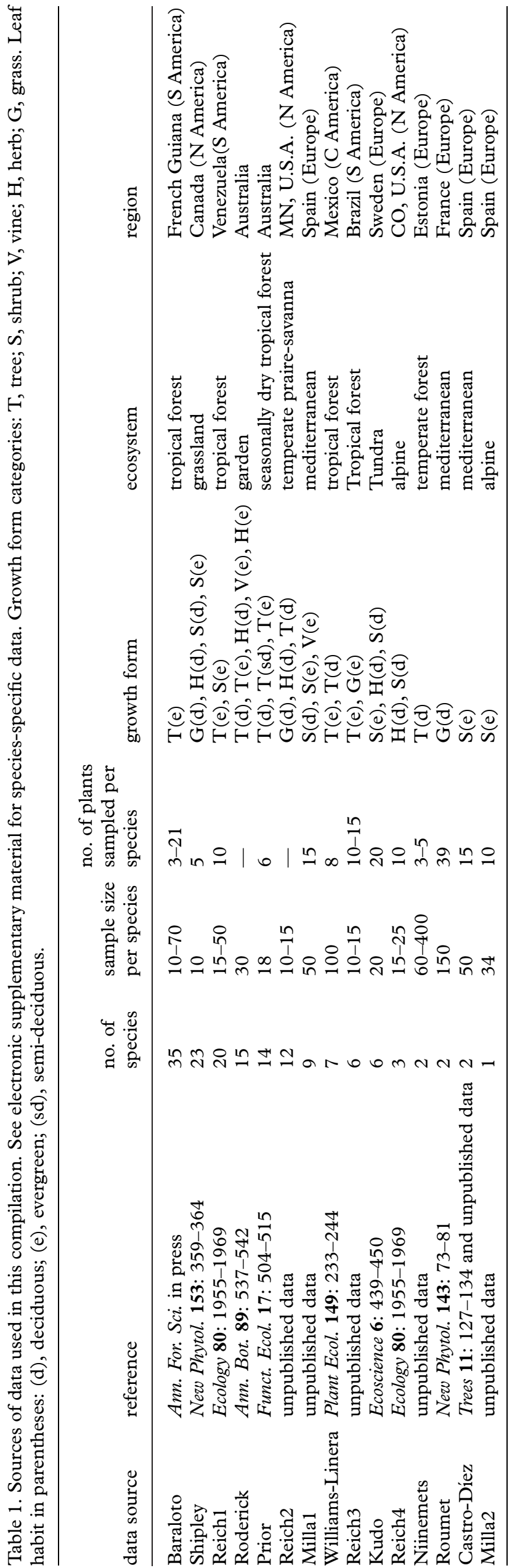

which may influence the scaling relations. In fact, several of the previous studies had contradictory results when mixing study scales: Shipley (1995) found that leaf mass scaled allometrically to leaf area when compared across species, but isometrically within species; Fonseca et al. (2000) reported that community-average leaf size-SLA relationships showed opposite trends than across-species patterns; and several papers highlight that SLA changes during plant ontogeny (Thomas \& Bazzaz 1999), which may in turn shift the SLA-leaf size allometry if leaf size did not change accordingly at the same rate and in the same direction than SLA (Sack et al. 2002).

Habitat, phylogeny and development may confound the characterization of the leaf size effect on SLA in broad interspecific comparisons. Therefore, we attempted to isolate putative confounding factors as outlined here and fully explained in the methods section. In short, we compiled data from sources where leaf dry mass (LM) and leaf surface area (LA) were measured in at least 10-15 independent replicates per species in a single environment (replicates came from a variable number of individual plants, depending on the original source, see table 1) and at a comparable developmental stage (i.e. fully expanded mature yearly leaves). Then we examined the scaling relationship of LM to LA within each of those species, evaluating $\mathrm{LM}=\alpha \mathrm{LA}^{\beta}$ by log-transforming this function, thus converting the power exponents to linear slope scores, to test the null hypothesis that $\beta=1$. The value of $\beta>1$ indicates that larger leaves have lower SLA, whereas $\beta<1$ means the opposite. If LA and LM scale isometrically, then changes in leaf size have no impact on SLA. We gathered a large data compilation of 157 species, expanding the scope of inference from previous intraspecific studies typically made with far fewer species.

\section{MATERIAL AND METHODS}

\section{(a) Compilation and filtering of data}

We aimed to isolate as far as possible the effect of changing leaf size from other confounding factors. To do so, we searched for published papers in which LA and LM were measured independently in at least $10-15$ replicates (leaves) per species, per developmental stage of leaves and plants, per canopy position and per location or experimental unit in experimental-gradient studies. We only considered studies of adult plants (no seedlings or saplings). Leaf ontogeny was standardized by including only fully mature current-year leaves. Also, for each individual species, data came from leaves in similar light environs and from a single site or plant community. This allowed us to calculate the scaling exponent of $\mathrm{LM}=\alpha \mathrm{LA}^{\beta}$ for each species separately. In this way, variation in factors such as differences between species or environment was kept as low as possible. This procedure constrained the number of data sources suitable for the compilation, since most multi-species studies had to be discarded due to low sample size per species.

Original, raw data for each measured leaf were obtained from the corresponding authors of published reports, or otherwise were gathered from our own databases or those of colleagues (table 1). When an original database supplied data for the same species in different environmental situations, we extracted the data subset that came from the control treatment in an experiment, the most typical situation in a gradient study, or an arbitrarily chosen condition (e.g. sun 
leaves instead of shade leaves). However, in case the fit of the regression equations of LM to LA was not tight enough to fit our criteria below, we selected the treatment level or location that yielded the best goodness of fit.

Initially, we compiled data for 194 species from 18 original sources. To obtain a reliable set of $\beta$, this initial database was purged so that all species surpass the following filtering criteria: (i) to be included in the final compilation, any species must show a statistically significant relation between (log)LA and $(\log ) \mathrm{LM}$, and the goodness of fit of that relation should be higher than $r^{2}=0.80$ and (ii) the range of LA variation should be at least twofold within each species. After applying the above filters, our final compilation comprised 6334 leaves of 157 species coming from 15 original sources (table 1 ).

\section{(b) Calculations and statistics}

Leaf mass (LM, g) and projected one-sided leaf surface area (LA, $\mathrm{cm}^{2}$ ) were assumed to be related to each other as defined by the following power function:

$$
\mathrm{LM}=\alpha \mathrm{LA}^{\beta} .
$$

To facilitate computation and visualization, we log-linearized the above function, thus converting the power exponents to linear slope scores

$\log (\mathrm{LM})=\log (\alpha)+\beta \log (\mathrm{LA})$.

In figure 1 , it is shown how a given trend on a LA-SLA scatter plot (figure $1 a$ ) translates into a (log)LA versus $(\log$ ) LM slope $(\beta)$ (figure $1 b$ ). Basically, $\beta>1$ indicates that SLA decreases as LA increases, whereas $\beta<1$ means the opposite. If LA and LM scale isometrically, then changes in leaf size have no impact on SLA.

To calculate slopes and intercepts of $(\log )$ LA versus ( $\log$ )LM, we used type II linear regression procedures. Type I (least squares) regression calculates regression parameters so that error in the dependent $(y)$ variable is minimized. This assumes no measurement error in the independent $(x)$ variable. Type II, or reduced major axis (RMA), regression minimizes both the error associated to variation in dependent and independent variables. The RMA regression is more appropriate when there is no a priori functional relation of dependency between $y$ and $x$ variables, and when the $x$ variable is subjected to measurement errors (Sokal \& Rohlf 1995). We fitted a RMA regression line to the $(\log )$ LM versus $(\log )$ LA data separately for each species. Also, for each species, a likelihood ratio test was run to evaluate whether $\beta$ of the RMA line was significantly higher, equal or lower than 1 (Warton $\&$ Weber 2002). Moreover, to test the initial hypothesis that $\beta=1$ in the complete dataset, we conducted a one sample $t$-test of the RMA $\beta$ set against $\beta=1$. Given the nature of our hypothesis regarding allocation of mass to mechanical support, we assess LM as a function of LA, but results can easily be examined in the opposite relationship (Niklas et al. 2007). Type II regression procedures were carried out using SMATR (v. I, Falster, D. S., Warton, D. I. \& Wright, I. J. http://www.bio.mq.edu.au/ ecology/SMATR). Detailed description of the methods for assessing whether $\beta$ varies with phylogenetic history, plant life form, or ecosystem of origin are provided in the electronic supplementary material.
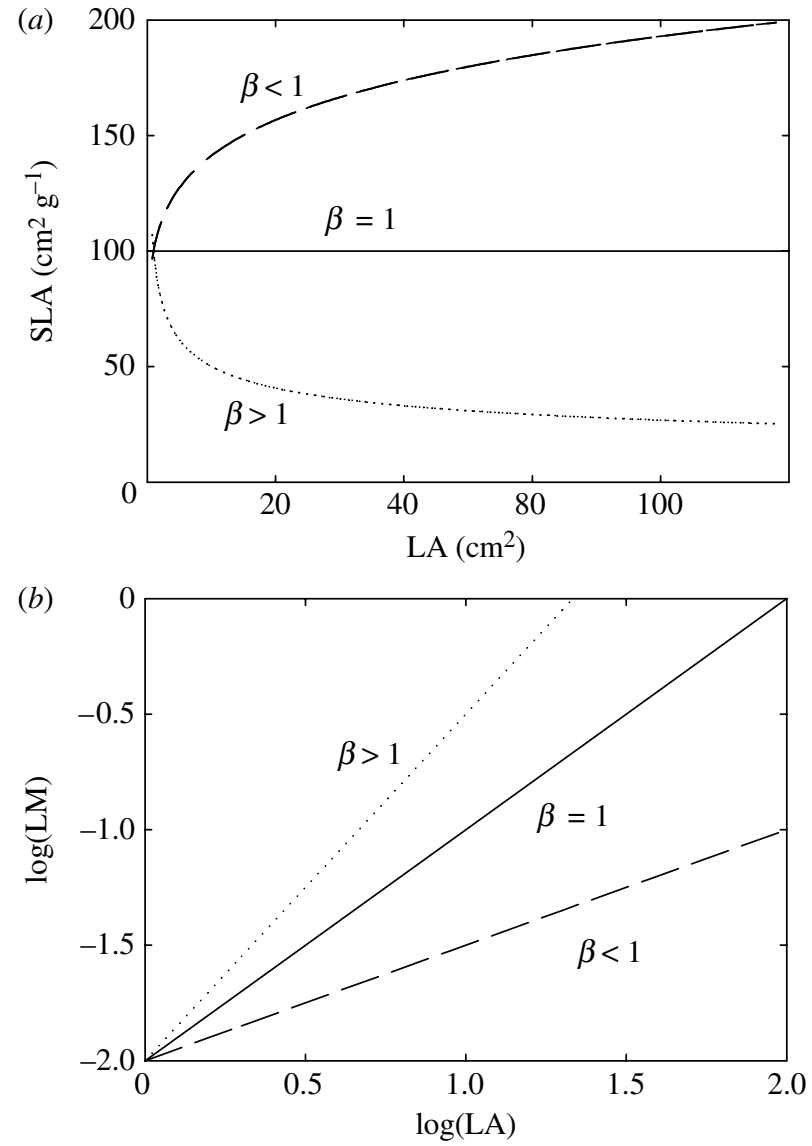

Figure 1. (a) Theoretical relationships between leaf area (LA, $\mathrm{cm}^{2}$ ) and specific leaf area (SLA, $\mathrm{cm}^{2} \mathrm{~g}^{-1}$ ) assuming three scenarios: (i) SLA is unaffected by leaf size, (ii) SLA increases as leaf size increases, and (iii) SLA decreases as leaf size increases. (b) Reflection of the scenarios depicted in (a) to the scaling of LA to leaf mass (LM, g). $\beta=$ exponent of the power function $\mathrm{LM}=\alpha \mathrm{LA}^{\beta}$. See text for further explanations.

\section{RESULTS}

Our 6334 leaves, 157 species dataset covered a range of LA from 0.03 (the smallest leaf of Empetrum hermaphroditum) to $1872 \mathrm{~cm}^{2}$ (the largest leaf of Sterculia pruriens). This spans six orders of magnitude and includes most of leaf size variation on Earth. The compilation included representatives of most plant growth form types and major biomes on Earth.

On average, the exponent $\beta$ for the power function $\mathrm{LM}=$ $\alpha \mathrm{LA}^{\beta}$ averaged 1.10 for the 157 species (figure 2). This average for $\beta$ was significantly higher than 1 in a one-sample $t$-test $(p<0.001$ ), with the $95 \%$ CI for $\beta$ of $1.08-1.13$. Thus, LM tended to increase proportionally faster than LA with each order of magnitude increase in both. Consequently, larger leaves showed lower SLA, on average.

The numerical difference between 1.10 and 1.00 may appear small. To show graphically how small differences in $\beta$ can translate into large differences in SLA when leaves differ in size, we modelled the rate of variation in $\mathrm{LM}$ as a function of LA as the point derivative of LM on LA $\left(\delta \mathrm{LM} / \delta \mathrm{LA}=(\mathrm{a} \beta) \mathrm{LA}^{(\beta-1)}\right)$ for each leaf of the compilation, assuming $\beta=1.10$, and $\mathrm{LA}=\mathrm{LA}$ of the actual leaf. $\delta \mathrm{LM} / \delta \mathrm{LA}$ reflects the instantaneous rate of increase in $\mathrm{LM}$ at a given value of LA. In figure 3, we plot $\delta \mathrm{LM} / \delta \mathrm{LA}$ for the whole range of leaf sizes of the study. It can be appreciated how the rate of LM increase per unit increase in leaf area is higher in larger-leaved species. 


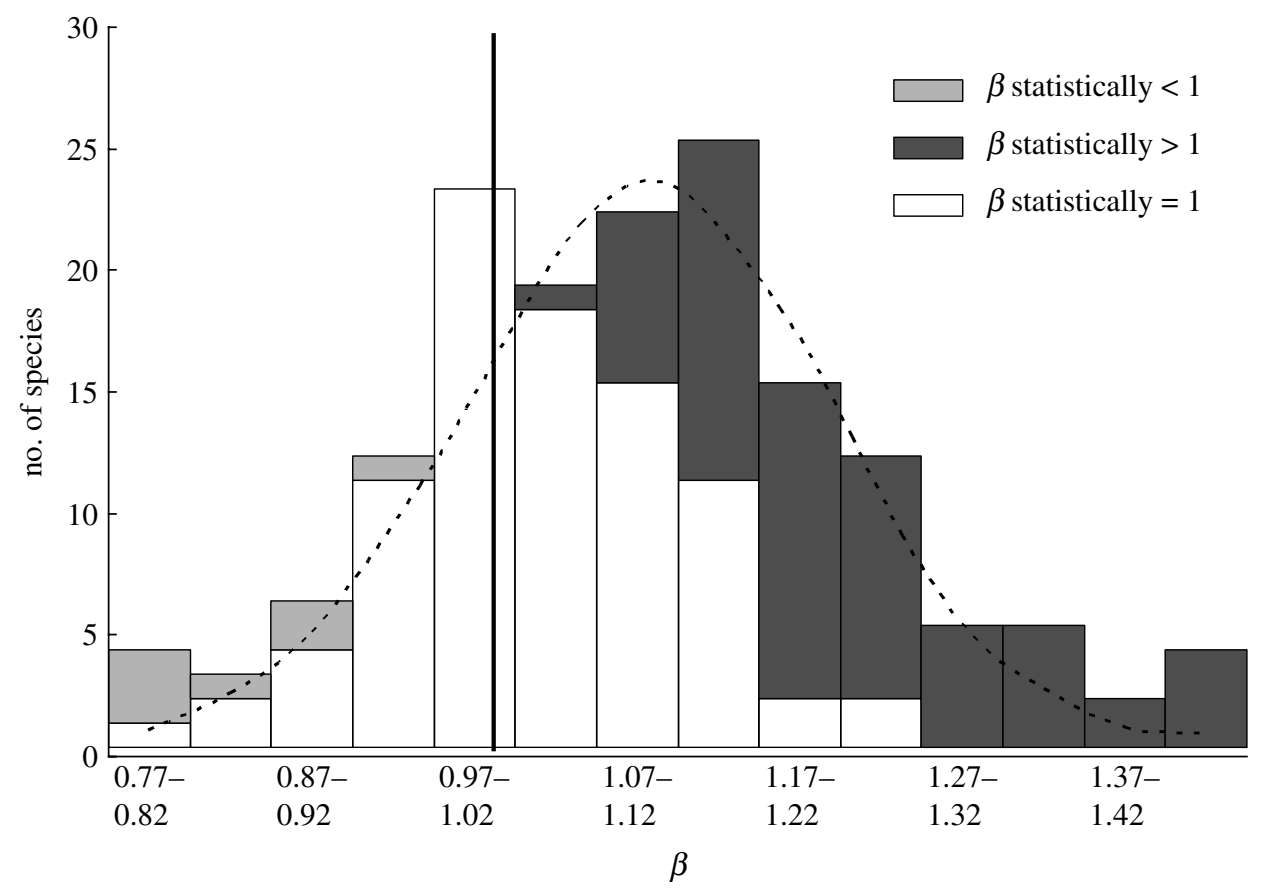

Figure 2. Frequency distribution of the power exponents of $\mathrm{LM}=\alpha \mathrm{LA}^{\beta}$ encountered in the 157 species of this compilation. Different patterns represent the statistical significance of the likelihood ratio test against $\beta=1$ for each species. Reference line: $\beta=1$.

However, it is equally remarkable that $\beta$ showed a great degree of variability among the species, ranging from 0.776 to 1.764 . Of the 157 species, 120 yielded a $\beta$ score higher than 1 , with 61 of those having a statistically higher $\beta$ when tested separately against $\beta=1$ (i.e. lower limit of $95 \%$ CI higher than 1), compared with 89 species that were not statistically different than 1 or 7 with $\beta$ significantly less than 1 (figure 2). Thus, the tendency for SLA to decrease with increasing leaf size is strong, but not obligate.

The tendency of phylogenetically related organisms to resemble each other did not translate in this study to similar $\beta$ scores for related species and/or clades. Treewide conservatism of $\beta$ scores was not significant ( $p>0.05$ for each of the fully resolved 50 trees, results not shown). Moreover, node-level analysis revealed that only 5 out of 94 bifurcating nodes showed significant conservatism of $\beta$ scores at $p=0.05$ (results not shown). We did not find any trend towards significant conservatism in older compared to younger nodes, as shown in figure 4 (no regression model fitted the scatter), which also suggests no major influence of phylogenetic inertia on $\beta$ scores of the study species. Thus, overall, the influence of phylogeny on the slope of LM to LA was negligible.

The graphs in figure 5 of the electronic supplementary material show how several characteristics of the raw data and of the (log)LM versus (log)LA regression fit affected: (i) the $\beta$ score obtained for each species, and (ii) the probability of $\beta$ being significantly different from 1 . First, $\beta$ was neither related to the number of replicates used to infer each species-specific RMA equation nor was it to the range of leaf sizes within a given species (figure $5 a, b$ electronic supplementary material). Additionally, we tested three statistical factors for possible role in causing species' $\beta$ values to differ significantly from 1: (i) the number of paired-data used to calculate the RMA equation, (ii) the range of variation in the arbitrarily considered independent variable (LA), and (iii) the goodness of fit of the RMA equation to the data. Overall, however, none of those

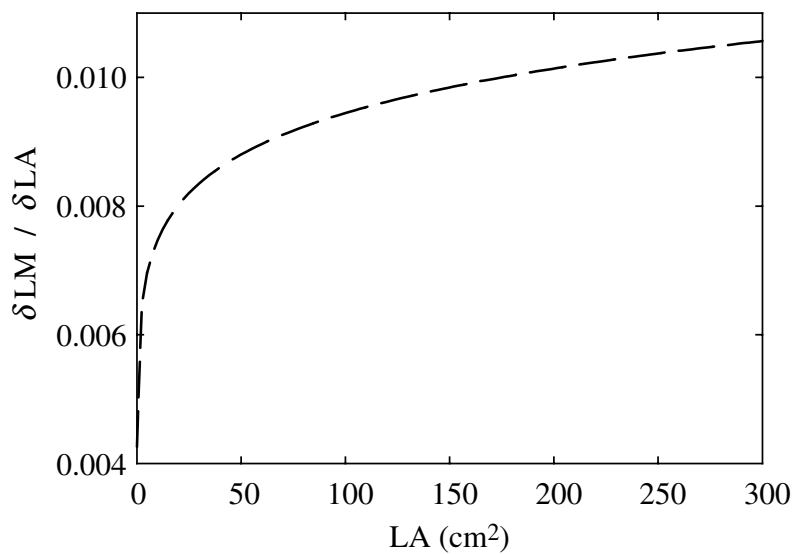

Figure 3. Instantaneous rate of change of $\mathrm{LM}$ with respect to increasing LA $(\delta \mathrm{LM} / \delta \mathrm{LA})$ at different ranges of LA. This theoretical relation assumes $\beta=1.10$ ( $\beta$ average of the 157 species studied here).

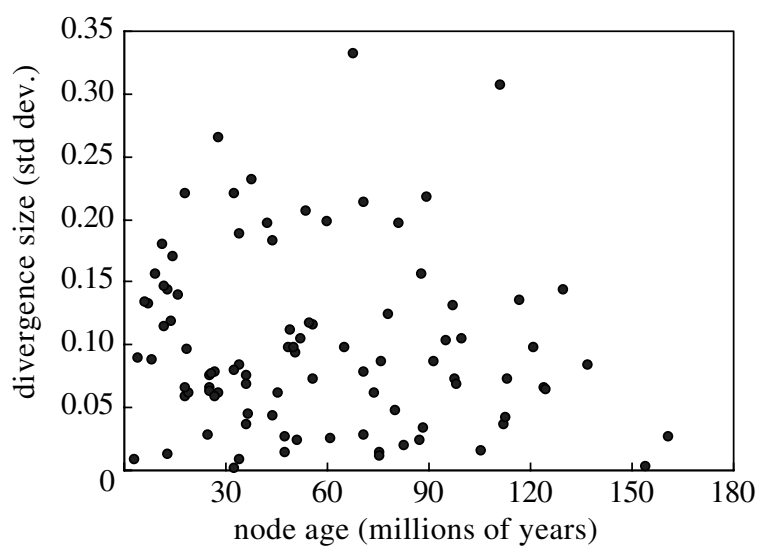

Figure 4. Divergence size of $\beta$ scores (standard deviation of subtended nodes) as a function of node age of the phylogenetic tree of the seed plants, pruned for the study species. No simple regression model explained a significant fraction of the scatter. (see electronic supplementary material for calculations) 
three factors affected the outcome of the statistical test (figure $5 c-e$ electronic supplementary material).

The scaling exponent, $\beta$, neither differed among growth forms $(p=0.59$, not shown) nor between plants showing deciduous, evergreen or semi-deciduous leaf habit $(p=0.45$, not shown), or between woody and herbaceous species $(p=0.73$, not shown). Also, $\beta$ did not differ among species from different ecosystems ( $p=0.38$, not shown). Furthermore, we neither found statistically significant relationships, nor trends, between $\beta$ and either mean annual temperature or annual rainfall at the study sites (data compiled from papers, or alternatively from national weather services, not shown). Also, we attempted several multiple regression and discriminant MANOVA techniques using the above plant and site traits as factors and $\beta$ as the dependent variable, but no significant trend arose (not shown).

\section{DISCUSSION}

Averaging across the 157 species examined here, leaf mass scales disproportionately faster than LA. Large leaves of a given species tend to have higher mass per unit leaf area than small leaves of the same species, for the majority of species. Since LM is a power function of LA, the allometric effect varies at different ranges of leaf size. For instance, for two species sharing the same scaling exponent $(\beta=1.07)$ but differing in average leaf size, the allometric effect differs in magnitude. A change from a small $96 \mathrm{~cm}^{2}$ leaf of Eucalyptus tetrodonta to a large $622 \mathrm{~cm}^{2}$ of the same species corresponds to a $22 \%$ decrease in SLA. In contrast, Quercus coccifera, which is on average small leaved, also experiences a decrease of SLA with increasing leaf size, though to a lesser extent (9\%). The increase with leaf size in leaf mass invested per unit leaf area corresponds directly to increased construction and maintenance costs of displaying a unit of leaf area. Construction costs (energy units) per unit leaf area are directly related to the mass invested per unit leaf area; for instance, construction costs per unit leaf area were strongly correlated and proportionally scaled $\approx 1: 1$ with the $g$ of dry mass per unit leaf area, based on a re-analysis $\left(\log \left(\mathrm{g}\right.\right.$ glucose per $\left.\mathrm{m}^{2}\right)=0.0876+1.04587(\log (1 / \mathrm{SLA}))$; $\left.n=121, r^{2}=0.98, p<0.001\right)$ of data for 121 plant species (cf. Villar \& Merino 2001). Maintenance costs (based on dark respiration rates per unit leaf area) increase linearly with dry mass per unit leaf area within each of 11 plant species (similar re-analysis of data from Lusk \& Reich 2000), as is generally the case. Hence, the increase in mass per unit leaf area of larger than smaller leaves directly translates into a greater cost to build and maintain a unit of leaf area, which, all else being equal, should constrain the maximum leaf size displayed.

Our $\beta=1.10$, central tendency at the within-species level, is generally in accordance with broad among-species trends worldwide (Niklas et al. 2007) and more limited intraspecific studies of approximately 20 species (Niklas et al. 2007; Price \& Enquist 2007). In a comparison of mean values for 1943 species, Niklas et al. (2007) found that overall, large leaves deploy less light-absorbing area per unit of dry mass investment than small leaves, and that additionally suggests that larger leaves require comparatively more nutrients per unit dry mass and thus probably allocate more nutrients to structural support rather than productive tissues. However, the tendency towards low leaf area per unit dry mass in larger leaves was much more muted in the large interspecific comparison (slope of 0.98 for $(\log ) \mathrm{LA}$ versus $(\log ) \mathrm{LM}$, equivalent to a slope of 1.02 for (log)LM versus (log)LA, as done in our study), and was not observed at all for trees, as a subset of the overall dataset. Hence, the tendency towards diminished leaf area display per $g$ investment in larger leaves is more pronounced as a phenotypic response within species than as a genotypic pattern among species. This is not necessarily surprising, as interspecific patterns (as in Niklas et al. 2007) could result entirely from inverse selection pressures for leaf size and SLA, and would not require any intrinsic phenotypic allometry of LM to LA. Our intraspecific results, therefore, suggest that there are biophysical and physiological consequences of increasing leaf size that are independent of current interspecific differences in multiple leaf trait strategies that may have arisen from selection in the past. Taken together, both reports (Niklas et al. 2007 and our current paper) support the idea of 'diminishing return' on mass investment with increasing leaf size using light capture potential as a measure of potential return, due to increased requirement for costly material support for a given leaf area with increasing leaf size.

At this point, it is important to highlight that, in spite of the $\beta=1.10$ central tendency, we found a remarkable range of scaling exponents (from $\beta=1.764$ to $\beta=0.776$ ) among the 157 study species. Thus, the size-dependent proportional shift in SLA across the observed range of leaf sizes (SLA VAR $_{\text {, }}$ see electronic supplementary material), although generally smaller than 1 (average $=0.87$ ), is higher than 1 for a fair amount of species. Surprisingly, this variation was unrelated to leaf habit, growth form, ecosystem of origin or climate, which are typical drivers of differences in plant traits (Wright et al. 2004). Additionally, the range of the LA and SLA spectra that each species occupies was not related to variation in $\beta$ among species (analyses not shown). It is also important to reflect on the consequences of changing LA on SLA from a quantitative viewpoint. Tripling LA for the leaves of a given species, and assuming $\beta=1.10$, produces a $10 \%$ decrease in SLA. Small as it may be, this drop in SLA increases constructions costs at the leaf level, which, scaled to the whole-plant level (i.e. multiplied per the number of leaves produced by an individual plant), should imply a significant increase in the pool of resources required to build light capturing leaf area. In conclusion, we found that a given species could possess almost any kind of LM-LA relationship. Nevertheless, there was a significant central tendency for larger leaves to show lower SLA. Hence, more often than not, the biomass (and related construction and maintenance) costs of deploying light-absorbing leaf area are higher for larger than for small leaves.

We profusely thank all researchers who kindly provided raw data from their publications or unpublished material (Christopher Baraloto, Bill Shipley, Ülo Niinemets, Lynda Prior, Guadalupe Williams-Linera, Michael Roderick, Catherine Roumet, Gaku Kudo and Pilar Castro-Díez), and to those who also sent data which, unfortunately, did not go into the final compilation after applying some filtering to the original dataset (Michael Morecroft, Diane Rowland, Jacek Oleksyn and Susan Cordell). We also thank SMATR and PHylocom developers for making their products free software, Jeannine Cavender-Bares and Rubén Torices for assistance in phylogenetic analyses and Christopher Baraloto 
and Lawren Sack for their in-depth reviews of earlier manuscripts. R.M. was supported by the Minisiterio de Educación y Ciencia (Spain) through a postdoctoral fellowship and a Juan de la Cierva contract. This research was supported by the National Science Foundation (NSF, USA) LongTerm Ecological Research (DEB-0080382) programme.

\section{REFERENCES}

Ackerly, D. D. \& Reich, P. B. 1999 Convergence and correlations among leaf size and function in seed plants: a comparative test using independent contrasts. $\mathrm{Am}$. f. Bot. 86, 1272-1281. (doi:10.2307/2656775)

Cornelissen, J. H. C., Pérez-Harguindeguy, N., Díaz, S., Grime, J. P., Marzano, B., Cabido, M., Vendramini, F. \& Cerabolini, B. 1999 Leaf structure and defence control litter decomposition rate across species and life forms in regional floras on two continents. New Phytol. 143, 191-200. (doi:10.1046/j.1469-8137.1999.00430.x)

Enquist, B. J. \& Niklas, K. J. 2002 Global allocation rules for patterns of biomass partitioning in seed plants. Science 295, 1517-1520. (doi:10.1126/science.1066360)

Fonseca, C. R., Overton, J. M., Collins, B. \& Westoby, M. 2000 Shifts in trait-combinations along rainfall and phosphorus gradients. F. Ecol. 88, 964-977. (doi:10. 1046/j.1365-2745.2000.00506.x)

Givnish, T. J. 1979 On the adaptive significance of leaf form. In Topics in plant population biology (eds O. T. Solbrig, S. Jain, G. B. Johnson \& P. H. Raven), pp. 375-407. New York, NY: Columbia University Press.

Givnish, T. J. 1987 Comparative studies of leaf form: assessing the relative roles of selective pressures and phylogenetic constraints. New Phytol. 106, 131-160.

Grubb, P. J. 1998 A reassessment of the strategies of plants which cope with shortages of resources. Persp. Plant Ecol. Evol. Syst. 1, 3-31. (doi:10.1078/1433-831900049)

Gunn, S., Farrar, J. F., Collis, B. E. \& Nason, M. 1999 Specific leaf area in barley: individual leaves versus whole plants. New Phytol. 143, 45-51. (doi:10.1046/j.1469-8137. 1999.00434.x)

Herrera, C. M. 2002 Correlated evolution of fruit and leaf size in bird-dispersed plants: species-level variance in fruit traits explained a bit further? Oikos 97, 426-432. (doi:10. 1034/j.1600-0706.2002.970312.x)

Howland, H. C. 1962 Structural, hydraulic, and 'economic' aspects of leaf venation and shape. In Biological prototypes and synthetic systems (eds E. E. Bernard \& M. R. Kare), pp. 183-191. New York, NY: Plenum Press.

Koch, G. W., Sillett, S. C., Jennings, G. M. \& Davis, S. D. 2004 The limits to tree height. Nature 428, 851-854. (doi:10.1038/nature02417)

Leonard, W. R. \& Robertson, M. L. 1994 Evolutionary perspectives on human nutrition: The influence of brain and body size on diet and metabolism. Am. F. Human Biol. 6, 77-88. (doi:10.1002/ajhb.1310060111)

Lusk, C. H. \& Reich, P. B. 2000 Relationships of leaf dark respiration with light environment and tissue nitrogen content in juveniles of 11 cold-temperate tree species. Oecologia 123, 318-329. (doi:10.1007/s004420051018)

Niinemets, Ü. \& Kull, O. 1994 Leaf weight per area and leaf size of 85 Estonian woody species in relation to shade tolerance and light availability. For. Ecol. Manage. 70, 1-10. (doi:10.1016/0378-1127(94)90070-1)
Niinemets, Ü. \& Kull, O. 1999 Biomass investment in leaf lamina versus lamina support in relation to growth irradiance and leaf size in temperate deciduous trees. Tree Physiol. 19, 349-358.

Niinemets, Ü., Portsmuth, A. \& Tobias, M. 2006 Leaf size modifies support biomass distribution among stems, petioles and mid-ribs in temperate plants. New Phytol. 171, 91-104. (doi:10.1111/j.1469-8137.2006.01741.x)

Niklas, K. J. 1999 Research review: a mechanical perspective on foliage leaf form and function. New Phytol. 143, 19-31. (doi:10.1046/j.1469-8137.1999.00441.x)

Niklas, K. J., Cobb, E. D., Niinemets, Ü., Reich, P. B., Sellin, A., Shipley, B. \& Wright, I. J. 2007 "Diminishing returns" in the scaling of functional leaf traits across and within species groups. Proc. Natl Acad. Sci. USA 104, 8891-8896. (doi:10.1073/pnas.0701135104)

Peters, H. R. 1983 The ecological implications of body size. New York, NY: Cambridge University Press.

Price, C. A. \& Enquist, B. J. 2007 Scaling mass and morphology in leaves: an extension of the WBE model. Ecology 88, 1132-1141.

Reich, P. B., Walters, M. B. \& Ellsworth, D. S. 1997 From tropics to tundra: global convergence in plant functioning. Proc. Natl Acad. Sci. USA 94, 13730-13734. (doi:10. 1073/pnas.94.25.13730)

Reich, P. B., Ellsworth, D. S. \& Walters, M. B. 1998 Leaf structure (specific leaf area) modulates photosynthesisnitrogen relations: evidence from within and across species and functional groups. Funct. Ecol. 12, 948-958. (doi:10. 1046/j.1365-2435.1998.00274.x)

Sack, L., Maranon, T. \& Grubb, P. J. 2002 Global allocation rules for patterns of biomass partitioning. Science 296, 1923. (doi:10.1126/science.296.5575.1923a)

Shipley, B. 1995 Structured interspecific determinants of specific leaf area in 34 species of herbaceous angiosperms. Funct. Ecol. 9, 312-319. (doi:10.2307/2390579)

Shipley, B., Vile, D. \& Garnier, E. 2006 From plant traits to plant communities: a statistical mechanistic approach. Science 314, 812-814. (doi:10.1126/science.1131344)

Sokal, R. R. \& Rohlf, F. J. 1995 Biometry. New York, NY: Freeman and Company.

Thomas, S. C. \& Bazzaz, F. A. 1999 Asymptotic height as a predictor of photosynthetic characteristics in Malaysian rain forest trees. Ecology 80, 1607-1622. (doi:10.2307/ 176550)

Villar, R. \& Merino, J. 2001 Comparison of leaf construction costs in woody species with differing leaf life-spans in contrasting ecosystems. New Phytol. 151, 213-226. (doi:10.1046/j.1469-8137.2001.00147.x)

Warton, D. I. \& Weber, N. C. 2002 Common slope tests for bivariate structural relationships. Biometric F. 44, 161-174. (doi:10.1002/1521-4036(200203)44:2<161::AID-BIMJ $161>3.0 . \mathrm{CO} ; 2-\mathrm{N}$ )

Westoby, M., Warton, D. \& Reich, P. B. 2000 The time value of leaf area. Am. Nat. 155, 649-656. (doi:10.1086/ 303346)

Wright, I. J. et al. 2004 The worldwide leaf economics spectrum. Nature 428, 821-827. (doi:10.1038/nature02403)

Wright, I. J. et al. 2005 Modulation of leaf economic traits and trait relationships by climate. Global Ecol. Biog. 14, 411-421. (doi:10.1111/j.1466-822x.2005.00172.x) 


\section{NOTICE OF CORRECTION}

The second equation in $\S 2 b$ is now presented in the correct form. 